\title{
GROUND STATES FOR THE PRESCRIBED MEAN CURVATURE EQUATION
}

\author{
L. A. PELETIER AND J. SERRIN
}

\begin{abstract}
We discuss the existence of radial ground states of problem (I), under appropriate restrictions on the bifurcation parameter $\lambda$ and the exponent $q$.
\end{abstract}

1. Introduction. We discuss the existence of radial solutions of the problem

$$
\begin{aligned}
& \operatorname{div}\left(\frac{\nabla u}{\sqrt{1+|\nabla u|^{2}}}\right)+f(u)=0 \text { in } \mathbf{R}^{n}, \\
& u(x) \rightarrow 0 \text { as } x \rightarrow \infty
\end{aligned}
$$

in which the function $f$ has the canonical behavior

$$
f(u)=-\lambda u+u^{q} .
$$

Here $n>1, q>1, \lambda>0$, and $\nabla u$ denotes the gradient of $u$.

Radial solutions of equation (1) have been studied in connection with the analysis of capillary surfaces $[\mathbf{4}, \mathbf{6}, \mathbf{9}]$. In that context, however, the source term $f$ was given by

$$
f(u)=\kappa u, \quad \kappa>0,
$$

and the interest was focused on singular solutions. In this note, to the contrary, we shall be concerned with regular positive solutions of problem (I), often referred to as ground states. For the related equation $\Delta u+f(u)=0$ such solutions have been extensively studied, and results about existence, nonexistence, and uniqueness have been obtained for different classes of functions $f$ (see for example $[\mathbf{1}, \mathbf{2}, \mathbf{3}, \mathbf{1 0}, \mathbf{1 1}$, 14, 15]).

For problem (I), with $f$ given by (3), it has recently been shown that when $\lambda>0$ there exist no ground states if either one of the following conditions holds:

$$
\begin{aligned}
\lambda & >\left(2 \frac{q+1}{q-1}\right)^{(q-1) /(q+1)} \quad[8] \\
q & \geq \frac{n+2}{n-2} \quad \text { when } n>2 \quad[\mathbf{1 2}]
\end{aligned}
$$

On the other hand, numerical work carried out by Evers and Levine [5] suggests that, for $\lambda$ small enough and $q<(n+2) /(n-2)$, problem (1)-(3) does indeed have a solution. In this paper we shall give an existence theorem which confirms this.

Received by the editors May 2, 1986.

1980 Mathematics Subject Classification (1985 Revision). Primary 53A10; Secondary 35B32. 
THEOREM. Suppose $q>1$, and additionally $q<(n+2) /(n-2)$ when $n>2$. Then there exists a number $\lambda_{0}>0$, depending only on $q$ and $n$, such that for every $\lambda<\lambda_{0}$ problem (1)-(3) has a positive radial solution.

When $\lambda \leq 0$, the situation is somewhat simpler. In particular, no ground states whatsoever can exist when $\lambda<0$ (the proof being essentially the same as in Lemma 3 below). When $\lambda=0$ and $q<n /(n-2)$ again no ground state exists, while for $\lambda=0$ and $q \geq(n+2) /(n-2)$ there are an infinite number of ground states (see [13]).

2. Proof of the Theorem. As a first step we observe that if $u(x)$ is a solution of $(1)-(3)$, then the function

$$
v(x)=u_{\lambda}(x)=\lambda^{-1 / 2 q} u\left(\lambda^{-(q-1) / 4 q} x\right)
$$

is a solution of the problem

$$
\begin{gathered}
\operatorname{div}\left(\frac{\nabla v}{\sqrt{1+\varepsilon|\nabla v|^{2}}}\right)+f_{\varepsilon}(v)=0 \text { in } \mathbf{R}^{n} \\
v(x) \rightarrow 0 \text { as } x \rightarrow \infty
\end{gathered}
$$

where

$$
\varepsilon=\lambda^{(q+1) / 2 q} \text { and } f_{\varepsilon}(v)=-\varepsilon v+v^{q} .
$$

On the other hand, if $v$ is a solution of (5)-(7) then (4) yields a solution of (1)-(3).

Note that if we set $\varepsilon=0$ in (5), we obtain the equation

$$
\Delta v+v^{q}=0 .
$$

Thus, for small $\varepsilon,(5)$ can formally be regarded as a perturbation of (8). For this reason, in the sequel we shall work with the equivalent problem (5), (6).

Let $v(r)$ be a positive radial solution of (5), (6). Then

$$
\begin{aligned}
& \left(\frac{v^{\prime}}{\sqrt{1+\varepsilon v^{\prime 2}}}\right)^{\prime}+\frac{n-1}{r} \frac{v^{\prime}}{\sqrt{1+\varepsilon v^{\prime 2}}}+f_{\varepsilon}(v)=0, \quad r>0 \\
& v^{\prime}(0)=0, \quad v(r) \rightarrow \infty \quad \text { as } r \rightarrow \infty
\end{aligned}
$$

Following [3], we use a shooting argument to prove the existence of a positive solution of problem (II). Thus we consider solutions $v(r, \xi)$ of $(9)$ which satisfy the initial conditions

$$
v(0)=\xi>0, \quad v^{\prime}(0)=0 .
$$

For small values of $r$, the existence and uniqueness of $v(r, \xi)$ is ensured by standard arguments. The following lemma, which is essentially taken from [8], gives conditions under which we can continue $v(\cdot, \xi)$ as long as both $v>0$ and $v^{\prime}<0$.

Let $\alpha$ denote the positive root of $f_{\varepsilon}(v)=0$; thus $\alpha=\varepsilon^{1 /(q-1)}$. In what follows we shall always suppose that

$$
0<\varepsilon<1
$$

so that also $\alpha<1$. 
LEMMA 1. Suppose (11) holds and that

$$
\alpha<\xi \leq 1 \text {. }
$$

Then $v(r, \xi)$ can be continued until either $v(r)$ or $v^{\prime}(r)$ reaches zero.

Proof. In view of $(12)$ we have $v^{\prime \prime}(0, \xi)<0$, whence $v^{\prime}(r, \xi)<0$ for small positive values of $r$. In turn $0<v \leq \xi$ for small values of $r$, so it is enough to show that $v^{\prime}$ does not reach $-\infty$ before either $v$ or $v^{\prime}$ vanishes.

We now put

$$
\begin{aligned}
& A(p)=\left(1+\varepsilon p^{2}\right)^{-1 / 2}, \quad E(p)=\frac{d}{d p}\left(\frac{p}{\left(1+\varepsilon p^{2}\right)^{1 / 2}}\right), \\
& H(p)=\int_{0}^{p} \rho E(\rho) d \rho=\frac{1}{\varepsilon}\left(1-\frac{1}{\left(1+\varepsilon p^{2}\right)^{1 / 2}}\right)
\end{aligned}
$$

and note that $H(p)>0$ for $p>0$. Then, if we multiply (9) by $v^{\prime}$ and integrate over $(0, r)$, we obtain

$$
H\left(\left|v^{\prime}(r)\right|\right)+(n-1) \int_{0}^{r} A\left(\left|v^{\prime}(\rho)\right|\right) v^{2}(\rho) \frac{d \rho}{\rho}=F(\xi)-F(v(r)),
$$

where $F(u)=\int_{0}^{u} f_{\varepsilon}(s) d s$. The function $F$ reaches its minimum value at $\alpha$. Hence

$$
F(\xi)-F(v(r)) \leq F(\xi)-F(\alpha) .
$$

Therefore, if

$$
F(1)-F(\alpha) \leq H(\infty)
$$

the identity (13) implies that $v^{\prime}(r)$ cannot reach $-\infty$.

For the specific choice of $A$ and $F$ made here, (14) becomes

$$
-\frac{\varepsilon}{2}+\frac{1}{q+1}+\frac{q-1}{2(q+1)} \varepsilon^{(q+1) /(q-1)} \leq \frac{1}{\varepsilon} .
$$

For $0<\varepsilon<1$ the left-hand side is less than $1 /(q+1)$ and the right-hand side greater than 1 . Hence, by virtue of (11), the condition (14) is indeed satisfied, and so $v^{\prime}(r)$ cannot reach $-\infty$.

Now let

$$
\begin{array}{r}
I_{+}=\left\{\xi \in(\alpha, 1]: \text { there exists } R>0 \text { such that } v^{\prime}(R, \xi)=0\right. \\
\text { and } v(r, \xi)>0 \text { for } r \in[0, R]\}, \\
I_{-}=\{\xi \in(\alpha, c]: \text { there exists } R>0 \text { such that } v(R, \xi)=0 \\
\text { and } \left.v^{\prime}(r, \xi)<0 \text { for } r \in(0, R]\right\} .
\end{array}
$$

Obviously $I_{+}$and $I_{-}$are disjoint and open in $(\alpha, 1]$.

Suppose both $I_{+}$and $I_{-}$are nonempty. Then there must exist a number $\xi_{0} \in(\alpha, 1)$ which does not belong to either $I_{+}$or $I_{-}$. We shall show that the corresponding function $v\left(r, \xi_{0}\right)$ is a solution of problem (II), as is required. Indeed for this value of $\xi$ there holds

$$
0<v\left(r, \xi_{0}\right)<\xi_{0} \quad \text { and } v^{\prime}\left(r, \xi_{0}\right)<0 \text { for } r \in(0, \infty)
$$

Hence we can define

$$
l=\lim _{r \rightarrow \infty} v\left(r, \xi_{0}\right) \quad\left(0 \leq l<\xi_{0}\right)
$$


LEMMA 2. $l=0$.

ProOF. By letting $r \rightarrow \infty$ in (13) we deduce that

$$
\int_{0}^{\infty} A\left(\left|v^{\prime}(\rho)\right|\right) v^{\prime 2}(\rho) \frac{d \rho}{\rho}<\infty
$$

Hence, using (13) again, it is evident that $\lim _{r \rightarrow \infty} v^{\prime}\left(r, \xi_{0}\right)$ exists. In view of (15) or of (16), this limit necessarily must be zero.

If we now take the limit in (9) we obtain

$$
\lim _{r \rightarrow \infty} v^{\prime \prime}\left(r, \xi_{0}\right)=f_{\varepsilon}(l) .
$$

Because $v^{\prime}$ is bounded, it follows that $f_{\varepsilon}(l)=0$ and hence either $l=0$ or $l=\alpha$.

To exclude the possibility that $l=\alpha$ we use an argument from [13]. Thus suppose for contradiction that ${ }^{1}$

$$
v(r) \searrow \alpha \quad \text { as } r \rightarrow \infty .
$$

Remembering the definition of $A$, we can write (9) as

$$
\left(r^{n-1} A\left(\left|v^{\prime}\right|\right) v^{\prime}\right)^{\prime}+r^{n-1} f_{\varepsilon}(v)=0 .
$$

Since $v>\alpha$, it follows that $r^{n-1} A\left(\left|v^{\prime}\right|\right) v^{\prime}$ is decreasing. Hence there exist constants $r_{0}>0, \delta>0$ such that

$$
r^{n-1} A\left(\left|v^{\prime}(r)\right|\right) v^{\prime}(r)<-\delta \text { for } r>r_{0} .
$$

Since $A(p)<1$ this implies $r^{n-1} v^{\prime}(r)<-\delta$ for $r>r_{0}$ which, in turn, shows that

$$
v(r)>\alpha+\frac{\delta}{n-2} r^{2-n} \text { for } r>r_{0} .
$$

(The case $n=2$ is easily treated by a separate argument, omitted here.)

On the other hand, if we integrate $(17)$ over $(0, r)$ and use the condition $v^{\prime}(0)=0$, there results by the convexity of $f_{\varepsilon}$

$$
\begin{aligned}
r^{n-1} A\left(\left|v^{\prime}(r)\right|\right) v^{\prime}(r) & =-\int_{0}^{r} s^{n-1} f_{\varepsilon}(v(s)) d s \\
& <-f_{\varepsilon}^{\prime}(\alpha) \int_{0}^{r} s^{n-1}\{v(s)-\alpha\} d s<-\Lambda r^{n}\{v(r)-\alpha\},
\end{aligned}
$$

where $\Lambda=f_{\varepsilon}^{\prime}(\alpha) / n=(q-1) \varepsilon>0$. In the last step note that $v(s)>v(r)$ for $s<r$.

Now $0<A(p)<1$ so that, in turn,

$$
v^{\prime}(r)<-\Lambda r\{v(r)-\alpha\} .
$$

Hence by integration $v(r)-\alpha<C e^{-\Lambda r^{2} / 2}$ for some positive constant $C$. This contradicts the lower bound (18). Therefore $l \neq \alpha$, and the proof is complete.

Since $l=0$, it follows that $v\left(r, \xi_{0}\right)$ is a positive solution of problem (II) as required.

\footnotetext{
${ }^{1}$ For simplicity, we shall occasionally write $v(r)$ rather than $v\left(r, \xi_{0}\right)$.
} 
It remains to prove that both $I_{+}$and $I_{-}$are nonempty for $\varepsilon$ small enough. We shall do this in the next two lemmas.

LEMMA 3. I $I_{+}$is nonempty.

We give two proofs, the first emphasizing a connection with oscillatory solutions of the Bessel equation, the second using (13) and energy ideas.

FIRST PROOF. We shall show that $\xi \in I_{+}$whenever $\xi-\alpha$ is small.

Note that $v(r, \alpha)=\alpha$ for all $r>0$ by the uniqueness of the initial value problem. Hence $v(r, \xi) \rightarrow \alpha$ and $v^{\prime}(r, \xi) \rightarrow 0$ as $\xi \rightarrow \alpha$, uniformly on compact subsets of $[0, \infty)$. Now consider the function

$$
w(r)=\frac{v(r, \xi)-\alpha}{\xi-\alpha}, \quad \xi \neq \alpha .
$$

Plainly, $w$ is a solution of the problem

$$
\left\{\begin{array}{l}
w^{\prime \prime}+\left(1+\varepsilon v^{2}\right) \frac{n-1}{r} w^{\prime}+\left(1+\varepsilon v^{2}\right)^{3 / 2} \frac{f_{\varepsilon}(v)}{v-\alpha} w=0 \\
w(0)=1, \quad w^{\prime}(0)=0
\end{array}\right.
$$

As $\xi \rightarrow \alpha$, the solutions $w$ tend uniformly on compact subsets of $[0, \infty)$ to the solution $W$ of the problem

$$
W^{\prime \prime}+\frac{n-1}{r} W^{\prime}+f_{\varepsilon}^{\prime}(\alpha) W=0, \quad W(0)=1, \quad W^{\prime}(0)=0
$$

where $f_{\varepsilon}^{\prime}(\alpha)=(q-1) \varepsilon>0$. This is a standard Bessel equation of the oscillatory type, and consequently $W$ is an oscillatory function. Since $w$ is uniformly near $W$ on compact subsets of $[0, \infty)$ for $\xi$ near $\alpha$, it follows that $w$ must be oscillatory as well, and so in turn $v$ must oscillate about $\alpha$. This implies that $\xi \in I_{+}$.

SECOND PROOF. Consider values of $\xi$ in the interval $(\alpha, \beta]$, where

$$
\beta=\left\{\frac{1}{2}(q+1) \varepsilon\right\}^{1 /(q-1)}
$$

has the property that $F(\beta)=0$ and $F(u)<0$ for $u \in(0, \beta)$. If $\xi$ is not in $I^{+}$, then there exists an $R \leq \infty$ such that $v(r, \xi)$ is decreasing on $(0, R)$ and

$$
\lim _{r \rightarrow R} v(r, \xi)=0 \text {. }
$$

This is obvious if $\xi \in I^{-}$. On the other hand, if $\xi$ is neither in $I^{+}$nor $I^{-}$, then we have $R=\infty$, and $l=0$ by Lemma 2 .

Now letting $r \rightarrow R$ in (13) we obtain a contradiction since

$$
F(v(r))-F(\xi) \rightarrow-F(\xi) \leq 0 .
$$

This completes the proof.

LEMMA 4. There exists a positive number $\varepsilon_{0}<1$ such that $I_{-}$is nonempty if $0<\varepsilon<\varepsilon_{0}$.

ProOF. If we set $\varepsilon=0$ in (9), we obtain

$$
v^{\prime \prime}+\frac{n-1}{r} v^{\prime}+v^{q}=0 .
$$


Let $v_{0}(r)$ be the solution of $(20)$ with initial values

$$
v(0)=1, \quad v^{\prime}(0)=0 .
$$

Then, because we have assumed that $q<(n+2) /(n-2)$,

$$
r_{0}=\sup \left\{r>0: v_{0}>0 \text { on }[0, r)\right\}<\infty
$$

and $v^{\prime}\left(r_{0}\right)<0$. This result is well known and is essentially due to Fowler [7]. For a direct proof see $[13$, Theorem 3.1].

Because (9) is a regular perturbation of (20), the solution $v_{\varepsilon}$ of (9) with initial values (21) has the property that $v_{\varepsilon} \rightarrow v_{0}$ as $\varepsilon \rightarrow 0$, uniformly on compact subsets of $[0, \infty)$. This means that there exists a number $\varepsilon_{0}, 0<\varepsilon_{0}<1$, such that

$$
r_{\varepsilon}=\sup \left\{r>0: v_{\varepsilon}>0 \text { on }[0, r)\right\}<\infty,
$$

when $0<\varepsilon<\varepsilon_{0}$. Thus $1 \in I_{-}$when $\varepsilon \in\left(0, \varepsilon_{0}\right)$.

With Lemma 4 we have shown that problem (II) has a positive solution, provided $\varepsilon<\varepsilon_{0}$. This implies that problem (I) has a positive radial solution if

$$
\lambda<\lambda_{0}=\varepsilon_{0}^{2 q /(q+1)} .
$$

Since we have studied solutions of problem (II) we can, if we wish, think of $n$ as a real parameter greater than 1 , rather than as an integral dimension number. It is also worth noting that any radial ground state for problem (I), in particular that constructed here, is monotonically decreasing in the variable $r$ (see [8]).

\section{REFERENCES}

1. F. V. Atkinson and L. A. Peletier, Ground states of $-\Delta u=f(u)$ and the Emden-Fowler equation, Arch. Rational Mech. Anal. 93 (1986), 103-127.

2. H. Berestycki and P.-L. Lions, Nonlinear scalar field equations. I, Arch. Rational Mech. Anal. 82 (1983), 313-345.

3. H. Berestycki, P.-L. Lions, and L. A. Peletier, An ODE approach to the existence of positive solutions for semilinear problems in $\mathbf{R}^{N}$, Indiana Univ. Math. J. 30 (1981), 141-157.

4. P. Concus and R. Finn, A singular solution of the capillary equation. I, II, Invent. Math. 29 (1975), 143-148, 149-159.

5. T. K. Evers, Numerical search for ground state solutions of a modified capillary equation, M. Sc. Thesis, Dept. of Math., Iowa State Univ., 1985.

6. R. Finn, On the behaviour of a capillary surface near a singular point, J. Analyse Math. 30 (1976), 156-163.

7. R. H. Fowler, Further studies of Emden's and similar differential equations, Quart. J. Math. Oxford Ser. (2) 2 (1931), 259-288.

8. B. Franchi, E. Lanconelli, and J. Serrin, Esistenza e unicitá degli stati fondamentali per equazoni ellitici quasilineari, Rend. Accad. Naz. Lincei. (in press).

9. W. E. Johnson and L. M. Perko, Interior and exterior boundary value problems from the theory of the capillary tube, Arch. Rational Mech. Anal. 29 (1968), 129-143.

10. K. McLeod and J. Serrin, Uniqueness of the ground state solution for $\Delta u+f(u)=0$, Proc. Nat. Acad. Sci. U.S.A. 78 (1981), 6592-6595.

11. Z. Nehari, On a non-linear differential equation arising in nuclear physics, Proc. Roy. Irish Acad. 62 (1963), 117-135.

12. W.-M. Ni and J. Serrin, Non-existence theorems for quasilinear partial differential equations, Rend. Circ. Mat. Palermo (2) 5 (1986).

13. __ Existence and nonexistence theorems for ground states of quasilinear partial differential equations. The anomalous case, Accad. Naz. Lincei. (in press). 
14. L. A. Peletier and J. Serrin, Uniqueness of positive solutions of semilinear equations in $\mathbf{R}^{n}$, Arch. Rational Mech. Anal. 81 (1983), 181-197.

15. __ Uniqueness of non-negative solutions of semilinear equations in $\mathbf{R}^{n}, \mathrm{~J}$. Differential Equations 61 (1986), 380-397.

16. W. A. Strauss, Existence of solitary waves in higher dimensions, Comm. Math. Phys. 55 (1977), 149-162.

Mathematical Institute, University of Leiden, Leiden, The Netherlands

SChool of Mathematics, University of Minnesota, Minneapolis, Minnesota 55455 